\title{
Existence of Solutions for Implicit Fractional Differential Systems with Coupled Nonlocal Conditions
}

\author{
Mengna Zhang ${ }^{1}$, Yansheng Liu ${ }^{2}$ \\ ${ }^{1}$ School of Mechanical Engineering, Shandong University, Jinan, 250061, P. R. China \\ ${ }^{2}$ Department of Mathematics, Shandong Normal University, Jinan, 250014, P. R. China \\ Email: 286560922@qq.com, yanshliu@gmail.com
}

\begin{abstract}
This article concerns the existence of solutions for implicit fractional differential systems with coupled nonlocal conditions of functional type. We use a vectorial version of Krasnoselskii's fixed point theorem in generalized metric space to overcome the lack of complete continuity of the associated integral operators. Moreover, the sufficient conditions for the existence will be weakened on the subinterval in which our nonlocal conditions act. An example is presented to illustrate the theory.
\end{abstract}

Keywords: Fractional differential systems, implicit differential equation, coupled nonlocal conditions, fixed point, vector-valued norm, spectral radius of a matrix.

\section{Introduction and Preliminaries}

In this article, we study the implicit fractional differential systems

$$
\left\{\begin{array}{l}
{ }^{C} D_{0^{+}}^{\beta} x_{1}(t)=f_{1}\left(t, x_{1}(t), x_{2}(t), \cdots, x_{n}(t)\right)+g_{1}\left(t,{ }^{C} D_{0^{+}}^{\beta} x_{1}(t), \cdots,{ }^{C} D_{0^{+}}^{\beta} x_{n}(t)\right) \\
{ }^{C} D_{0^{+}}^{\beta} x_{2}(t)=f_{2}\left(t, x_{1}(t), x_{2}(t), \cdots, x_{n}(t)\right)+g_{2}\left(t,{ }^{C} D_{0^{+}}^{\beta} x_{1}(t), \cdots,{ }^{C} D_{0^{+}}^{\beta} x_{n}(t)\right) \\
\cdots \\
{ }^{C} D_{0^{+}}^{\beta} x_{n}(t)=f_{n}\left(t, x_{1}(t), x_{2}(t), \cdots, x_{n}(t)\right)+g_{n}\left(t,{ }^{C} D_{0^{+}}^{\beta} x_{1}(t), \cdots,{ }^{C} D_{0^{+}}^{\beta} x_{n}(t)\right)
\end{array}\right.
$$

for $t \in[0,1]$, subject to the coupled nonlocal conditions

$$
\left\{\begin{array}{l}
x_{1}(0)=\alpha_{11}\left[x_{1}\right]+\alpha_{12}\left[x_{2}\right]+\ldots+\alpha_{1 n}\left[x_{n}\right] \\
x_{2}(0)=\alpha_{21}\left[x_{1}\right]+\alpha_{22}\left[x_{2}\right]+\ldots+\alpha_{2 n}\left[x_{n}\right] \\
\ldots \\
x_{n}(0)=\alpha_{n 1}\left[x_{1}\right]+\alpha_{n 2}\left[x_{2}\right]+\ldots+\alpha_{n n}\left[x_{n}\right]
\end{array}\right.
$$

where $f_{i}, g_{i}:[0,1] \times R^{n} \rightarrow R(i=1,2, \cdots, n)$ are continuous functions, $\alpha_{i j}: C[0,1] \rightarrow R(i, j=1,2, \cdots, n)$ are continuous linear functionals, ${ }^{C} D_{0^{+}}^{\beta}$ is the Caputo fractional derivative of order $0<\beta \leq 1$.

It is well known that fractional derivatives provide a more excellent tool for the description of memory and hereditary properties of various materials and processes than integer derivatives in various fields of applied sciences and engineering ([1-6]). Recently, considerable attention has been given to a special class of fractional differential equations, that is, implicit fractional differential equations ([7-11]). For example, in [7], the authors considered the following initial value problem of implicit fractional order differential equation

$$
\left\{\begin{array}{l}
{ }^{C} D^{\alpha} y(t)=f\left(t, y(t),{ }^{C} D^{\alpha} y(t)\right), \quad t \in[0, b], \quad b>0 \\
y(0)=y_{0}
\end{array}\right.
$$


where $0<\alpha \leq 1, f:[0, b] \times R \times R \rightarrow R$ is a given function. By using fixed point theory and approximation method, some existence and uniqueness results were obtained.

In [8], by using Banach contraction principle, Schauder's fixed point theorem and nonlinear alternative of Leray-Schauder type, the authors investigated the following boundary value problem of the form

$$
\left\{\begin{array}{l}
{ }^{C} D^{\alpha} y(t)=f\left(t, y(t),{ }^{C} D^{\alpha} y(t)\right), \quad t \in[0, T], \quad T>0 \\
y(0)=y_{0}, y(T)=y_{1}
\end{array}\right.
$$

where $1<\alpha \leq 2, y_{0}, y_{1} \in R, f:[0, T] \times R \times R \rightarrow R$ is a given function.

On the other hand, nonlocal problems for differential equations were extensively discussed in the literature by various methods (see [14-19, 22, 23]). For instance, in [14], the authors deal with the existence, localization and multiplicity of positive solutions to nonlocal problems for first order differential systems

$$
\left\{\begin{array}{l}
u^{\prime}=f(t, u), \quad t \in[0,1] \\
u(0)=\alpha[u]
\end{array}\right.
$$

where $f:[0,1] \times R_{+}^{n} \rightarrow R^{n}$ is a continuous function and $\alpha: C\left([0,1], R^{n}\right) \rightarrow R^{n}$ is a linear continuous operator.

In [18], the authors considered the following first order differential systems

$$
\left\{\begin{array}{l}
u_{1}^{\prime}(t)=f_{1}\left(t, u_{1}(t), u_{2}(t), \cdots, u_{n}(t)\right) \\
u_{2}^{\prime}(t)=f_{2}\left(t, u_{1}(t), u_{2}(t), \cdots, u_{n}(t)\right) \\
\cdots \\
u_{n}^{\prime}(t)=f_{n}\left(t, u_{1}(t), u_{2}(t), \cdots, u_{n}(t)\right)
\end{array}\right.
$$

for a.e. $t \in[0,1]$, subject to the coupled nonlocal conditions

$$
\left\{\begin{array}{l}
u_{1}(0)=\alpha_{11}\left[u_{1}\right]+\alpha_{12}\left[u_{2}\right]+\ldots+\alpha_{1 n}\left[u_{n}\right] \\
u_{2}(0)=\alpha_{21}\left[u_{1}\right]+\alpha_{22}\left[u_{2}\right]+\ldots+\alpha_{2 n}\left[u_{n}\right] \\
\ldots \\
u_{n}(0)=\alpha_{n 1}\left[u_{1}\right]+\alpha_{n 2}\left[u_{2}\right]+\ldots+\alpha_{n n}\left[u_{n}\right]
\end{array}\right.
$$

where $f_{i}:[0,1] \times R^{n} \rightarrow R(i=1,2, \cdots, n)$ are $L^{1}$-Carathéodory functions, $\alpha_{i j}: C[0,1] \rightarrow R(i, j=1,2, \cdots, n)$ are continuous linear functionals.

Unfortunately, implicit integer order differential equations with nonlocal conditions, to our knowledge, were rarely investigated except for $[12,13]$. The paper [12] obtained an absolutely continuous solution for an implicit first order equation under nonlocal conditions, via Schauder's fixed point theorem and Kolmogorov's compactness criterion in $L^{1}$. In [13], the author used the Kransnoselskii's fixed point theorem for implicit first order systems with nonlocal conditions. As far as we know, there is no paper to studying implicit fractional order differential equations with nonlocal conditions. The purpose of this paper is to fill this gap and investigate (1.1) subject to the coupled nonlocal conditions (1.2). The main features of this paper are as follows. First, the equation considered here is an implicit fractional order differential equation and the boundary conditions are coupled nonlocal conditions. Second, the main tool used here is vectorial version of Krasnoselskii's fixed point theorem in generalized metric space to overcome the lack of complete continuity arising from implicit equations.

The paper is organized as follows. In the rest of this section, some preliminaries are stated. Section 2 contains the main results and their proof. Finally, in section 3, an example is worked out to demonstrate the main result.

For brevity, rewrite the problem (1.1) (1.2) into the vector form

$$
\left\{\begin{array}{l}
{ }^{C} D_{0^{+}}^{\beta} x(t)=f(t, x(t))+g\left(t,{ }^{C} D_{0^{+}}^{\beta} x(t)\right) \\
x(0)=\alpha[x]
\end{array}\right.
$$


where $x=\left(x_{1}, x_{2}, \cdots, x_{n}\right)^{T}, f=\left(f_{1}, f_{2}, \cdots, f_{n}\right)^{T}, g=\left(g_{1}, g_{2}, \cdots, g_{n}\right)^{T}$ and

$$
\begin{gathered}
\alpha[x]=\left(\alpha_{1}[x], \alpha_{2}[x], \cdots, \alpha_{n}[x]\right)^{T}, \\
\alpha_{i}[x]=\alpha_{i 1}\left[x_{1}\right]+\alpha_{i 2}\left[x_{2}\right]+\cdots+\alpha_{i n}\left[x_{n}\right], i=1,2, \cdots, n .
\end{gathered}
$$

Note that $\alpha$ is a continuous mapping from $C[0,1]^{n}$ to $R^{n}$.

Denote $u(t)={ }^{C} D_{0^{+}}^{\beta} x(t)$, we obtain

$$
x(t)=h(t)+x(0),
$$

where $x(0)=\left(x_{1}(0), x_{2}(0), \cdots, x_{n}(0)\right)^{T}$ and $h(t)=\int_{0}^{t} \frac{(t-s)^{\beta-1}}{\Gamma(\beta)} u(s) d s$. The condition $x(0)=\alpha[x]$ gives

$$
x(0)=\alpha[h]+\alpha[x(0)] .
$$

In view of (1.4) and (1.5), we obtain

$$
\alpha[x(0)]=\left(\alpha_{1}[x(0)], \alpha_{2}[x(0)], \cdots, \alpha_{n}[x(0)]\right)^{T}
$$

and

$$
\begin{aligned}
\alpha_{i}[x(0)] & =\alpha_{i 1}\left[x_{1}(0)\right]+\alpha_{i 2}\left[x_{2}(0)\right]+\cdots+\alpha_{i n}\left[x_{n}(0)\right] \\
& =x_{1}(0) \alpha_{i 1}[1]+x_{2}(0) \alpha_{i 2}[1]+\cdots+x_{n}(0) \alpha_{i n}[1] .
\end{aligned}
$$

Hence $\alpha[x(0)]=\alpha[1] x(0)$, where $\alpha[1]=\left(a_{i j}[1]\right)_{1 \leq i, j \leq n}$.

Here and through the paper, the vectors in $R^{n}$ are seen as column vectors.

Assume that $I-\alpha[1]$ is nonsingular, where $I$ is the unit matrix of order $n$. Then

$$
x(0)=(I-\alpha[1])^{-1} \alpha[h] .
$$

Therefore, $x(t)=(I-\alpha[1])^{-1} \alpha[h]+h(t)$. Let

$$
\begin{gathered}
(F u)(t)=f\left(t,(I-\alpha[1])^{-1} \alpha[h]+h(t)\right), \\
(G u)(t)=g(t, u(t)) .
\end{gathered}
$$

Then the problem (1.1) is equivalent to

$$
u=F u+G u .
$$

Let $T=F+G$, then $T: C[0,1]^{n} \rightarrow C[0,1]^{n}$. Therefore, the system (1.6) can be regarded as a fixed point problem for the operator $T$.

We now give some notations, definitions and basic results which are used in this paper. By a vectorvalued metric on a set $\mathrm{X}$ we mean a mapping $d: X \times X \rightarrow R^{n}$ such that

(1) $d(x, y) \geq 0$ for all $x, y \in X$ and $d(x, y)=0$ if and only if $x=y$;

(2) $d(x, y)=d(y, x)$ for all $x, y \in X$;

(3) $d(x, y) \leq d(x, z)+d(z, y)$ for all $x, y, z \in X$.

The meaning of " $\leq$ " is the natural componentwise order relation of $R^{n}$, namely, if $x, y \in R^{n}, x=$ $\left(x_{1}, x_{2}, \cdots, x_{n}\right)^{T}, y=\left(y_{1}, y_{2}, \cdots, y_{n}\right)^{T}$, by " $x \leq y$ " we mean $x_{i} \leq y_{i}$ for $i=1,2, \cdots, n$.

We call the pair $(X, d)$ a generalized metric space. For such a space, convergence and completeness are similar to those in usual metric spaces. An operator $T: X \rightarrow X$ is said to be a generalized contraction, if there exists a matrix $M$ with $\rho(M)<1$ such that

$$
d(T(x), T(y)) \leq M d(x, y)
$$

for all $x, y \in X$, where $\rho(M)$ is the spectral radius of $M$.

For any nonnegative square matrix $M$, the following properties are equivalent (see $[20-21]$ ):

(1) $\rho(M)<1$

(2) $M^{k} \rightarrow 0$ as $k \rightarrow \infty$;

(3) $I-M$ is nonsingular and $(I-M)^{-1}=I+M+M^{2}+\cdots$; 
(4) $I-M$ is nonsingular and $(I-M)^{-1}$ is a nonnegative matrix.

As we know, for both discrete and continuous nonlocal conditions, it's important to consider the interval on which the condition acts, namely, the support of the conditions. Throughout this paper, we assume that the support of the condition $x(0)=\alpha[x]$ is the subinterval $\left[0, t_{0}\right]$ of $[0,1]$, with the property $\alpha[x]=0$ whenever $x(t) \equiv 0$ on $\left[0, t_{0}\right]$.

Denote the vector-norm on $R^{n}$

$$
\|x\|=\left(\begin{array}{c}
\left|x_{1}\right| \\
\left|x_{2}\right| \\
\vdots \\
\left|x_{n}\right|
\end{array}\right)
$$

for $x \in R^{n}, x=\left(x_{1}, x_{2}, \cdots, x_{n}\right)^{T}$.

Denote by $M_{n \times n}(R), M_{n \times n}\left(R_{+}\right)$the sets of all square matrices of order $n$ with elements in $R, R_{+}$, respectively, and the inequality

$$
\|M x\| \leq|M|\|x\|
$$

also holds for $M=\left(a_{i j}\right)_{1 \leq i, j \leq n} \in M_{n \times n}(R),|M|=\left(\left|a_{i j}\right|\right)_{1 \leq i, j \leq n} \in M_{n \times n}\left(R_{+}\right)$, and $x \in R^{n}$.

Consider a special norm on $C[0,1]$,

$$
|x|_{C[0,1]}=\max \left\{|x|_{C\left[0, t_{0}\right]},|x|_{C\left[t_{0}, 1\right]}\right\},
$$

where $|x|_{C\left[0, t_{0}\right]}=\max _{t \in\left[0, t_{0}\right]}|x(t)|,|x|_{C\left[t_{0}, 1\right]}=\max _{t \in\left[t_{0}, 1\right]}|x(t)| e^{-\theta(t-\eta)}$ for given $\theta>0, \eta<t_{0}$ which will be specified later.

Note that $\|\alpha[x]\| \leq|\alpha|\|x\|_{C\left[0, t_{0}\right]}$, here $|\alpha|=\left(\left|\alpha_{i j}\right|\right)_{1 \leq i, j \leq n},\left|\alpha_{i j}\right|$ is the norm of the functional $\alpha_{i j}$.

Now we state the following lemma and theorem that will be used in the sequel.

Lemma 1.1. ([20]) If $A \in M_{n \times n}\left(R_{+}\right)$is a matrix with $\rho(A)<1$, then $\rho(A+B)<1$ for every matrix $B \in M_{n \times n}\left(R_{+}\right)$whose elements are small enough.

Theorem 1.2. ([21]) Let $(X,\|\cdot\|)$ be a generalized Banach space, D be a nonempty closed bounded convex subset of $X$ and $T: D \rightarrow X$ such that

(i) $T=F+G$ with $F: D \rightarrow X$ completely continuous and $G: D \rightarrow X$ a generalized contraction, i.e. there exists a matrix $M \in M_{n \times n}\left(R_{+}\right)$with $\rho(M)<1$, such that $\|G(x)-G(y)\| \leq M\|x-y\|$ for all $x, y \in D$;

(ii) $F(x)+G(y) \in D$ for all $x, y \in D$.

Then $T$ has at least one fixed point in D.

\section{$2 \quad$ Main Results}

In this section we assume that

$$
\left|f_{i}(t, u)\right| \leq\left\{\begin{array}{l}
a_{i 1}\left|u_{1}\right|+a_{i 2}\left|u_{2}\right|+\cdots+a_{i n}\left|u_{n}\right|+a_{i}, t \in\left[0, t_{0}\right] \\
b_{i 1}\left|u_{1}\right|+b_{i 2}\left|u_{2}\right|+\cdots+b_{i n}\left|u_{n}\right|+b_{i}, \quad t \in\left[t_{0}, 1\right]
\end{array}\right.
$$

for all $u \in R^{n}$,

$$
\left|g_{i}(t, u)-g_{i}(t, v)\right| \leq c_{i 1}\left|u_{1}-v_{1}\right|+c_{i 2}\left|u_{2}-v_{2}\right|+\cdots+c_{i n}\left|u_{n}-v_{n}\right|,
$$

for all $u, v \in R^{n}$ and $t \in[0,1]$. Here $a_{i j}, b_{i j}, c_{i j}, a_{i}, b_{i}(i, j=1,2, \cdots, n)$ are nonnegative numbers.

Rewrite (2.1) and (2.2) in the vector form

$$
\begin{gathered}
\|f(t, u)\| \leq\left\{\begin{array}{l}
A\|u\|+S, t \in\left[0, t_{0}\right] \\
B\|u\|+L, t \in\left[t_{0}, 1\right]
\end{array}\right. \\
\|g(t, u)-g(t, v)\| \leq C\|u-v\|
\end{gathered}
$$


where $A=\left(a_{i j}\right)_{1 \leq i, j \leq n}, B=\left(b_{i j}\right)_{1 \leq i, j \leq n}, C=\left(c_{i j}\right)_{1 \leq i, j \leq n} \in M_{n \times n}\left(R_{+}\right)$, and $S=\left(a_{1}, a_{2}, \cdots, a_{n}\right)^{T}$, $L=\left(b_{1}, b_{2}, \cdots, b_{n}\right)^{T} \in R_{+}^{n}$.

The following theorem is our main results.

Theorem 2.1. Assume that (2.1) and (2.2) hold. If the spectral radius of the matrix $M_{0}+C$ is less than one, then the problem (1.1) and (1.2) has at least one solution, where $M_{0}=\frac{t_{0}^{\beta}}{\beta \Gamma(\beta)} A\left(\left|(I-\alpha[1])^{-1}\right||\alpha|+I\right)$.

Proof. Firstly, the fact that $F$ is completely continuous follows in a routine way from Assoli-Arzelà theorem.

Secondly, it follows from (2.2) that

$$
\begin{aligned}
\|(G u)(t)-(G v)(t)\| & =\|g(t, u(t))-g(t, v(t))\| \\
& \leq C\|u(t)-v(t)\| \\
& \leq C\|u-v\|_{C\left[0, t_{0}\right]}, \quad t \in\left[0, t_{0}\right]
\end{aligned}
$$

for any $u, v \in C[0,1]^{n}$. Taking the supremum on $\left[0, t_{0}\right]$, one can obtain that

$$
\|G u-G v\|_{C\left[0, t_{0}\right]} \leq C\|u-v\|_{C\left[0, t_{0}\right]} .
$$

On the other hand, from (2.2) it follows that

$$
\begin{aligned}
\|(G u)(t)-(G v)(t)\| & =\|g(t, u(t))-g(t, v(t))\| \\
& \leq C\|u(t)-v(t)\| \\
& =e^{\theta(t-\eta)} e^{-\theta(t-\eta)} C\|u(t)-v(t)\| \\
& \leq e^{\theta(t-\eta)} C\|u(t)-v(t)\|_{C\left[t_{0}, 1\right]}, \quad t \in\left[0, t_{0}\right]
\end{aligned}
$$

for any $u, v \in C[0,1]^{n}$. Dividing by $e^{\theta(t-\eta)}$ and taking the supremum on $\left[t_{0}, 1\right]$, we obtain

$$
\|G u-G v\|_{C\left[t_{0}, 1\right]} \leq C\|u-v\|_{C\left[t_{0}, 1\right]} .
$$

From (2.3) and (2.4), it is easy to see that

$$
\|G u-G v\|_{C[0,1]} \leq C\|u-v\|_{C[0,1]} .
$$

According to $\rho\left(M_{0}+C\right)<1$ and $C<M_{0}+C$, we have $\rho(C)<1$. Hence $G$ is a generalized contraction.

Lastly, we look for a nonempty, bounded, closed and convex subset $D$ of $C[0,1]^{n}$ such that $F(D)+$ $G(D) \subset D$. In fact, for any $u \in C[0,1]^{n}, t \in\left[0, t_{0}\right]$, one can obtain that

$$
\begin{aligned}
\|h(t)\| & =\left\|\int_{0}^{t} \frac{(t-s)^{\beta-1}}{\Gamma(\beta)} u(s) d s\right\| \\
& \leq \int_{0}^{t} \frac{(t-s)^{\beta-1}}{\Gamma(\beta)}\|u(s)\| d s \\
& \leq \int_{0}^{t} \frac{(t-s)^{\beta-1}}{\Gamma(\beta)} d s\|u\|_{C\left[0, t_{0}\right]} \\
& =\frac{t^{\beta}}{\beta \Gamma(\beta)}\|u\|_{C\left[0, t_{0}\right]} \\
& \leq \frac{t_{0}^{\beta}}{\beta \Gamma(\beta)}\|u\|_{C\left[0, t_{0}\right]} .
\end{aligned}
$$


This together with (2.1) guarantees that

$$
\begin{aligned}
\|(F u)(t)\| & =\left\|f\left(t,(I-\alpha[1])^{-1} \alpha[h]+h(t)\right)\right\| \\
& \leq A\left\|(I-\alpha[1])^{-1} \alpha[h]+h(t)\right\|+S \\
& \leq M_{0}\|u\|_{C\left[0, t_{0}\right]}+S, t \in\left[0, t_{0}\right] .
\end{aligned}
$$

Taking the maximum on $\left[0, t_{0}\right]$, we obtain

$$
\|F u\|_{C\left[0, t_{0}\right]} \leq M_{0}\|u\|_{C\left[0, t_{0}\right]}+S .
$$

On the other hand, one can obtain that

$$
\begin{aligned}
&\left\|\int_{0}^{t_{0}} \frac{(t-s)^{\beta-1}}{\Gamma(\beta)} u(s) d s\right\| \leq \int_{0}^{t_{0}} \frac{(t-s)^{\beta-1}}{\Gamma(\beta)}\|u(s)\| d s \\
& \leq \int_{0}^{t_{0}} \frac{\left(t_{0}-s\right)^{\beta-1}}{\Gamma(\beta)}\|u(s)\| d s \\
& \leq \int_{0}^{t_{0}} \frac{\left(t_{0}-s\right)^{\beta-1}}{\Gamma(\beta)} d s\|u\|_{C\left[0, t_{0}\right]} \\
&=\frac{t_{0}^{\beta}}{\beta \Gamma(\beta)}\|u\|_{C\left[0, t_{0}\right]}, t \in\left[t_{0}, 1\right] \\
&\left\|\int_{t_{0}}^{t} \frac{(t-s)^{\beta-1}}{\Gamma(\beta)} u(s) d s\right\| \leq \int_{t_{0}}^{t} \frac{(t-s)^{\beta-1}}{\Gamma(\beta)}\|u(s)\| d s \\
&=\int_{t_{0}}^{t} \frac{(t-s)^{\beta-1}}{\Gamma(\beta)} e^{\theta(s-\eta)} e^{-\theta(s-\eta)}\|u(s)\| d s \\
& \leq \int_{t_{0}}^{t} \frac{(t-s)^{\beta-1}}{\Gamma(\beta)} e^{\theta(s-\eta)} d s\|u\|_{C\left[t_{0}, 1\right]} \\
&=\int_{0}^{t-t_{0}} \frac{\sigma^{\beta-1}}{\Gamma(\beta)} e^{-\theta \sigma} d \sigma e^{\theta(t-\eta)}\|u\|_{C\left[t_{0}, 1\right]} \\
&=\frac{1}{\theta^{\beta}} \int_{0}^{\theta\left(t-t_{0}\right)} \frac{\tau^{\beta-1}}{\Gamma(\beta)} e^{-\tau} d \tau e^{\theta(t-\eta)}\|u\|_{C\left[t_{0}, 1\right]} \\
& \leq \frac{1}{\theta^{\beta}} e^{\theta(t-\eta)}\|u\|_{C\left[t_{0}, 1\right]}, t \in\left[t_{0}, 1\right] . \\
&
\end{aligned}
$$

Then

$$
\begin{aligned}
\|(F u)(t)\| & \leq B\left\|(I-\alpha[1])^{-1} \alpha[h]+h(t)\right\|+L \\
& \leq B\left\|(I-\alpha[1])^{-1} \alpha[h]+\int_{0}^{t_{0}} \frac{(t-s)^{\beta-1}}{\Gamma(\beta)} u(s) d s\right\|+B\left\|\int_{t_{0}}^{t} \frac{(t-s)^{\beta-1}}{\Gamma(\beta)} u(s) d s\right\|+L \\
& \leq \frac{t_{0}^{\beta}}{\beta \Gamma(\beta)} B\left(\left|(I-\alpha[1])^{-1} \| \alpha\right|+I\right)\|u\|_{C\left[0, t_{0}\right]}+L+\frac{1}{\theta^{\beta}} e^{\theta(t-\eta)} B\|u\|_{C\left[t_{0}, 1\right]}, t \in\left[t_{0}, 1\right] .
\end{aligned}
$$

Dividing by $e^{\theta(t-\eta)}$ and taking the supremum on $\left[t_{0}, 1\right]$, we obtain

$$
\|F u\|_{C\left[t_{0}, 1\right]} \leq\left(\frac{t_{0}^{\beta}}{\beta \Gamma(\beta)} B\left(\left|(I-\alpha[1])^{-1} \| \alpha\right|+I\right)\|u\|_{C\left[0, t_{0}\right]}+L\right) e^{-\theta\left(t_{0}-\eta\right)}+\frac{1}{\theta^{\beta}} B\|u\|_{C\left[t_{0}, 1\right]} .
$$

Now taking advantage of the choice of $\eta<t_{0}$, we assume (choosing large enough $\theta>0$ ) that

$$
\begin{gathered}
e^{-\theta\left(t_{0}-\eta\right)} B\left(\left|(I-\alpha[1])^{-1}\right||\alpha|+I\right)\|u\|_{C\left[0, t_{0}\right]} \leq A\left(\left|(I-\alpha[1])^{-1} \| \alpha\right|+I\right)\|u\|_{C\left[0, t_{0}\right]}, \\
e^{-\theta\left(t_{0}-\eta\right)} L \leq S .
\end{gathered}
$$


By deduction, one can obtain that

$$
\|F u\|_{C\left[t_{0}, 1\right]} \leq M_{0}\|u\|_{C\left[0, t_{0}\right]}+S+\frac{1}{\theta^{\beta}} B\|u\|_{C\left[t_{0}, 1\right]} .
$$

Now, (2.6) and (2.7) imply that

$$
\|F u\|_{C[0,1]} \leq\left(M_{0}+\frac{1}{\theta^{\beta}} B\right)\|u\|_{C[0,1]}+S .
$$

On the other hand, it follows from (2.5) that

$$
\|G u\|_{C[0,1]} \leq C\|u\|_{C[0,1]}+P,
$$

for every $u \in C[0,1]^{n}$, where

$$
P=\|G(0)\|_{C[0,1]} .
$$

Now we look for $R=\left(R_{1}, R_{2}, \ldots, R_{n}\right) \in R_{+}^{n}$ such that $\|F u+G v\|_{C[0,1]} \leq R$ for $u, v \in C[0,1]^{n}$ with $\|u\|_{C[0,1]} \leq R$ and $\|v\|_{C[0,1]} \leq R$. To this end, according to (2.8) and (2.9), it is sufficient to show $\left(M_{0}+\frac{1}{\theta^{\beta}} B+C\right) R+S+P \leq R$. Equivalently

$$
S+P \leq\left(I-M_{0}-\frac{1}{\theta^{\alpha}} B-C\right) R .
$$

Since $\rho\left(\left(M_{0}+C\right)<1\right.$ and the entries of $\frac{1}{\theta^{\beta}} B$ are as small as desired for $\theta>0$ large enough, according to Lemma 1.1 , we can choose $\theta$ such that

$$
\rho\left(M_{0}+\frac{1}{\theta^{\beta}} B+C\right)<1 .
$$

Then, $I-M_{0}-\frac{1}{\theta^{\beta}} B-C$ is invertible and its inverse $\left(I-M_{0}-\frac{1}{\theta^{\beta}} B-C\right)^{-1}$ is a nonnegative matrix , (2.10) is equivalent to

$$
R \geq\left(I-M_{0}-\frac{1}{\theta^{\beta}} B-C\right)^{-1}(S+P) .
$$

Therefore, $F(D)+G(D) \subset D$ if we consider $D=\left\{u \in C[0,1]^{n}:\|u\|_{C[0,1]} \leq R\right\}$. Thus the problem (1.1) (1.2) have at least one solution.

\section{An Example}

Example 3.1. Consider the following nonlocal problem

$$
\left\{\begin{array}{l}
{ }^{C} D_{0^{+}}^{\frac{1}{2}} x(t)=\frac{1}{4} \sin x+\frac{1}{4} y+p(t)+\sin \left(\frac{\sqrt{2}}{2 \Gamma\left(\frac{1}{2}\right)}^{C} D_{0^{+}}^{\frac{1}{2}} x(t)+\frac{\sqrt{2}}{2 \Gamma\left(\frac{1}{2}\right)}^{C} D_{0^{+}}^{\frac{1}{2}} y(t)\right) \\
{ }^{C} D_{0^{+}}^{\frac{1}{2}} y(t)=\frac{1}{4} x+\frac{1}{4} \cos y+q(t)+\frac{\sqrt{2}}{2 \Gamma\left(\frac{1}{2}\right)} \cos \left({ }^{C} D_{0^{+}}^{\frac{1}{2}} x(t)\right)+\frac{\sqrt{2}}{2 \Gamma\left(\frac{1}{2}\right)} \cos \left({ }^{C} D_{0^{+}}^{\frac{1}{2}} y(t)\right) \\
x(0)=\int_{0}^{\frac{1}{2}} 2 x(s)+y(s) d s \\
y(0)=\int_{0}^{\frac{1}{2}} x(s)+2 y(s) d s
\end{array}\right.
$$

where $p, q \in C[0,1]$.

This problem can be regarded as the form of (1.1) (1.2). In this case,

$$
\begin{aligned}
& f_{1}(t, x, y)=\frac{1}{4} \sin x+\frac{1}{4} y+p(t), \\
& f_{2}(t, x, y)=\frac{1}{4} x+\frac{1}{4} \cos y+q(t), \\
& g_{1}(t, x, y)=\sin \left(\frac{\sqrt{2}}{2 \Gamma\left(\frac{1}{2}\right)} x+\frac{\sqrt{2}}{2 \Gamma\left(\frac{1}{2}\right)} y\right), \\
& g_{2}(t, x, y)=\frac{\sqrt{2}}{2 \Gamma\left(\frac{1}{2}\right)} \cos x+\frac{\sqrt{2}}{2 \Gamma\left(\frac{1}{2}\right)} \cos y .
\end{aligned}
$$


We have $t_{0}=\frac{1}{2}, \beta=\frac{1}{2}, n=2$ and $f_{1}, f_{2}$ satisfy $(2.1)$ with

$$
\begin{array}{ll}
a_{11}=b_{11}=\frac{1}{4}, & a_{1}=|p|_{C\left[0, \frac{1}{2}\right]}, \\
a_{12}=b_{12}=\frac{1}{4}, & b_{1}=|p|_{C\left[\frac{1}{2}, 1\right]}, \\
a_{21}=b_{21}=\frac{1}{4}, & a_{2}=|q|_{C\left[0, \frac{1}{2}\right]}, \\
a_{22}=b_{22}=\frac{1}{4}, & b_{2}=|q|_{C\left[\frac{1}{2}, 1\right]} .
\end{array}
$$

Also, $g_{1}, g_{2}$ satisfy $(2.2)$ with

$$
c_{11}=c_{12}=c_{21}=c_{22}=\frac{\sqrt{2}}{2 \Gamma\left(\frac{1}{2}\right)} .
$$

In addition,

$$
\begin{aligned}
& \alpha_{11}[1]=\left|\alpha_{11}\right|=1, \\
& \alpha_{12}[1]=\left|\alpha_{12}\right|=\frac{1}{2}, \\
& \alpha_{21}[1]=\left|\alpha_{21}\right|=\frac{1}{2}, \\
& \alpha_{22}[1]=\left|\alpha_{22}\right|=1 .
\end{aligned}
$$

Then

$$
\begin{gathered}
\alpha[1]=\left[\begin{array}{cc}
1 & \frac{1}{2} \\
\frac{1}{2} & 1
\end{array}\right], \quad|\alpha|=\left[\begin{array}{cc}
1 & \frac{1}{2} \\
\frac{1}{2} & 1
\end{array}\right], \\
\left|(I-\alpha[1])^{-1}\right||\alpha|+I=2 I, M_{0}=\frac{\sqrt{2}}{2 \Gamma\left(\frac{1}{2}\right)} I, M_{0}+C=\frac{\sqrt{2}}{\Gamma\left(\frac{1}{2}\right)} I .
\end{gathered}
$$

It is easy to see that $\rho\left(M_{0}+C\right)=\frac{\sqrt{2}}{\Gamma\left(\frac{1}{2}\right)}<1$. Therefore, according to Theorem 2.1 the problem (3.1) has at least one solution.

\section{References}

1. S. Abbes, M. Benchohra, G.M. N'Guérékata, Topics in Fractional Differential Equations, Springer-Verlag, New York, 2012.

2. G. A. Anastassiou, Advances on Fractional Inequalities, Springer, New York, 2011.

3. K. Diethelm, The Analysis of Fractional Differential Equations, Lecture Notes in Mathematics, 2010.

4. A.A. Kilbas, H.M. Srivastava, J.J. Trujillo, Theory and Applications of Fractional Differential Equations, North-Holland Mathematics Studies, Elsevier Science B.V. vol. 204, pp. 2453-2461, 2006.

5. A.M.A. El-Sayed, Fractional differential equations, Bull.Fac.Sci.Assiut Univ.A, vol. 16, pp. 271-275, 1987.

6. V.E. Tarasov, Fractional Dynamics: Application of Fractional Calculus to Dynamics of particles, Fields and Media, Springer, Heidelberg; Higher Education Press, Beijing, 2010.

7. J.J Nieto, A. Ouahab, V. Venktesh, Implicit fractional differential equations via the Liouville-Caputo derivative, Mathematics, vol. 3, pp. 398-411, 2015.

8. M. Benchohra, J.R. Graef, S. Hamani, "Existence results for boundary value problems with nonlinear fractional differential equations," Appl. Anal. vol. 87, pp. 851-863, 2008.

9. M. Benchohra, J.E. Lazreg, Nonlinear fractional implicit differential equations, Commun. Appl. Anal. vol. 17, pp. 471-482, 2013.

10. M. Benchohra, J.E. Lazreg, "Existence and uniqueness results for nonlinear implicit fractional differential equations with boundary conditions," Rom. J. Math. Comput. Sci. vol. 4, pp. 60-72, 2014.

11. A.N. Vityuk, A.V. Mikhailenko, "The Darboux problem for an implicit differential equation of fractional order," Ukr. Mat. Visn. vol. 7, pp. 439-452, 585, 2010.

12. A.M.E. El-Sayed, E.M. Hamdallah, K.W. Elkadeky, "Internal nonlocal and integral condition problems of the differential equation $x^{\prime}=f\left(t, x, x^{\prime}\right)$," Journal of Nonlinear Sciences \& Its Applications, vol. 3, pp. 193-199, 2011. 
13. O. Bolojan, "Implicit first order differential systems with nonlocal conditions," Electronic Journal of Qualitative Theory of Differential Equations, 2015, 2014.

14. D. Trif, "Multiple positive solutions of nonlocal initial value problems for first order differential systems," Nonlinear Analysis Theory Methods \& Applications, vol. 75, pp. 5961-5970, 2012.

15. O. Nica, "Initial-value problems for first-order differential systems with general nonlocal conditions," Electronic Journal of Differential Equations, vol. 2, pp. 1394-1396, 2012.

16. O. Nica, "Nonlocal initial value problems for first order differential systems," Fixed Point Theory, vol. 13, pp. 603-612, 2012.

17. O. Nica, "On the nonlocal initial value problem for first order differential systems," International Journal on Fixed Point Theory Computation \& Applications, vol. 56, pp. 113-125, 2011.

18. O. Nica, G. Infante, R. Precup, "Existence results for systems with coupled nonlocal initial conditions," Nonlinear Analysis Theory Methods \& Applications, vol. 94, pp. 231-242, 2014.

19. R. Precup, Methods in Nonlinear Integral Equations, Kluwer, Dordrecht, 2002.

20. R. Precup, "The role of matrices that are convergent to zero in the study of semilinear operator systems," Mathematical \& Computer Modelling, vol. 49, pp. 703-708, 2009.

21. A. Viorel, Contributions to the study of nonlinear evolution equations, Ph.D thesis, Babes-Bolyai University of Cluj-Napoca, 2011.

22. G. Infante, F.M. Minhlös, P. Pietramala, "Non-negative solutions of systems of ODEs with coupled boundary conditions," Commun. Nonlinear Sci. Numer. Simul. vol. 17, pp. 4952-4960, 2012.

23. C. Yuan, D. Jiang, D. OąŕRegan, R.P. Agarwal, "Multiple positive solutions to systems of nonlinear semipositone fractional differential equations with coupled boundary conditions," Electronic Journal of Qualitative Theory of Differential Equations, vol. 13, pp. 1-13, 2012. 